\title{
Sleeve Gastrectomy is the Most Common Cause of Gastro-Esophageal Reflux Disease in Comparison with Other Bariatric Operations
}

\author{
Ahmad Elnasasra ${ }^{2}$, Ahmad Jaber ${ }^{2}$, Hasan Kais ${ }^{2}$ and Midhat Abu Sneineh ${ }^{1 *}$ \\ ${ }^{1}$ Department of General Surgery, Bariatric Unit, AZ sint jan Medical Center, Belgium \\ ${ }^{2}$ Department of Surgery A, Shamir (Assaf Harofeh) Medical Center, Israel \\ *Corresponding author: Midhat Abu Sneineh, Department of General Surgery, Bariatric Unit, AZ sint jan Medical Center, Belgium
}

\section{ARTICLE INFO}

Received: 慧 November 05, 2020

Published: ${ }^{ \pm}$November 09, 2020

Citation: Ahmad Jaber, Ahmad Elnasasra, Hasan Kais, Midhat Abu Sneineh. Sleeve Gastrectomy is the Most Common Cause of Gastro-Esophageal Reflux Disease in Comparison with Other Bariatric Operations. Biomed J Sci \& Tech Res 31(5)2020. BJSTR. MS.ID.005153.

Keywords: GERD; Bariatric operations; Gastrectomy; Gastric Reflux; Bariatric Surgery

Abbreviations: GERD: Gastro-Esophageal Reflux Disease; RNYGB: Roux En Y Gastric Bypass; SG: Sleeve Gastrectomy; LAGB: Laparoscopic Adjustable Gastric Band; IHD: Ischemic Heart Disease; IBD: Inflammatory Bowel Disease; COPD: Chronic Obstructive Pulmonary Disease

\section{ABSTRACT}

Purpose: GERD is one of the complications of bariatric operations that my affect quality of life. Our aim is to perform a retrospective cohort study to determine the incidence of symptomatic GERD following different types of bariatric surgery and which operations are considered a contraindication of GERD. In addition, we are attempting to identify the risk factors of GERD after bariatric surgery.

Materials and Methods: Medical records of 729 patients hospitalized for bariatric surgery between January 2010 and June 2019 in Shamir (Assaf Harofeh) Medical Center were reviewed.

Results: There were significant difference between the type of the bariatric procedure and the incidence of GERD symptoms after the operation. The incidence of symptomatic GERD in patients who underwent SG was $39.9 \%$ ( $\mathrm{p}=0.0131$ ). This was significantly higher comparing to $16.4 \%$ following roux en y gastric bypass, $23.4 \%$ following LAGB, and 11\% following OAGB. 113 patients out of 718 had a positive swallow test and of these patient 71 developed GERD symptoms post-operatively without correlation to the degree of reflux at the swallow test but with statistically significant correlation to the type of operation especially for SG (P value $<0.001$ ) and to our knowledge this was never reported in the literature.

Conclusion: SG is a good bariatric procedure option but should be contraindicated in asymptomatic reflux contrast swallow study and symptomatic GERD patients preoperatively because of high levels of symptomatic GERD post-operatively. Asymptomatic reflux at contrast swallow study pre-operatively should be considered a risk factor for GERD after the operation.

\section{Introduction}

Bariatric operations are considered the best option for losing weight in morbidly obese patients, these operations include Sleeve Gastrectomy (SG), Roux En Y Gastric Bypass (RNYGB), One Anastomosis Gastric Bypass (OAGB) and Laparoscopic Adjustable Gastric Band (LAGB) [1,2], sleeve gastrectomy is one of the most common bariatric procedure worldwide, it is still considered a safe bariatric operation [3], epidemiological data shows that maintaining a normal BMI may reduce the likelihood of developing GastroEsophageal Reflux Disease (GERD) and its potential complications
[4], so bariatric operations if they decrease overall weight will lead to improvement in GERD symptoms but unfortunately that is not always the case. The causes of gastric reflux in general not fully understood but there are theories about abnormal relaxation of the lower esophageal sphincter, increased frequency of transient sphincter relaxation, or from increased pressure from the stomach secondary to a hiatus hernia or increased intra-abdominal pressure [5]. The literature suggests that different surgeries have different impacts on the symptomatology of GERD and that careful 
assessment may be needed before performing bariatric surgery in patients with GERD [6]. GERD as a complication after bariatric surgery ranges between mild that responds well to lifestyle modifications, to intermediate that improves with daily treatment with proton pump inhibitor, to devastating complication that may affect quality of life and will need surgical treatment, thus choosing the appropriate operation according to GERD symptoms prior to the surgery is an important step.

El-Hadi et al reported that SG has been associated with an increased incidence of GERD following the procedure, however gastric banding and RNYGB has been shown to improve the symptoms of GERD in the short-term but but a new reflux symptoms and esophagitis in the long-term results [7]. Currently the data about GERD after bariatric surgery and the impact of bariatric operations on GERD is scarce so our aim is to perform a retrospective cohort study to determine the incidence of symptomatic GERD following different types of bariatric surgery and which operations are considered a contraindication of GERD. In addition, we are attempting to identify the risk factors of GERD after bariatric surgery.

\section{Methods}

Medical records of 729 patients hospitalized for elective bariatric surgery between January 2010 and June 2019 at Shamir (Assaf Harofeh) Medical Center were reviewed. Twenty-one patients were excluded because of missing data. The study was approved by the Institutional Review Board/Ethics Committee of the Shamir (Assaf Harofeh) Medical Center. Ages from 18 until 65 years and both sexes included in the study. We collected data from outpatient clinic records and telephone interview questionnaire. Available variables included demographic information, age, gender, and primary and secondary diagnoses. Categorical variables were summarized with frequencies and percentages. Continuous variables were summarized using sample size, mean, standard deviation, median, minimum, maximum, and 95\% CI. Correlations between categorical variables and incidence of GERD after surgery were tested for significance using the chi-square test. Correlations between continuous variables and incidence of GERD after surgery were tested for significance using the independent t-test. Baseline characteristics of interest included sex, age, diagnosis of GERD, and Body Mass Index (BMI) group.

\section{Results}

We retrospectively evaluated 718 patients with an average follow-up of 24 months (range 12-36 months), we collected the information from outpatient clinic as well as telephone interview using (GERD-Q questionnaire) [8] as an assessment for GERD symptoms (Table 1). GerdQ is a self-administered 6-item questionnaire that was recently developed as a tool to improve and standardize symptom-based diagnosis and evaluation of treatment response in patients with GERD [9]. According to outpatient clinic records, 236 (32.9\%) patients have GERD symptoms (heartburn, regurgitation, dysphagia, odynophagia, increased salivation and chest pain) according to medical history before the operation (Table 2). Mean age was $48 \pm 19$ years (76\% females). There were 218 laparoscopic SG (30.4\%), 175 laparoscopic LAGB (24.4\%), 61 RYGB (8.5\%), and 264 (36.8\%), OAGB procedures during the study period (Table 2). At the follow-up period, 96 of 718 patients (\%13.4) developed new symptomatic GERD and 71 of 236 preoperative GERD patients (30\%) continued to have the same or worse GERD symptoms after the surgery (Table 3) so a total of 167 after the surgery for the period of the follow-up (Table 4).

Table 1: GerdQ self-assessment questionnaire.

\begin{tabular}{|c|c|c|c|c|c|}
\hline \multirow[t]{2}{*}{ S. No } & \multirow{2}{*}{$\begin{array}{c}\text { Symptoms in the previous week } \\
\text { Question: }\end{array}$} & \multicolumn{4}{|c|}{ Symptoms presence } \\
\hline & & 0 days & 1 day & 2-3 days & 4-7 days \\
\hline 1. & How often did you have a burning feeling behind your breastbone (heartburn)? & 0 & 1 & 2 & 3 \\
\hline 2. & $\begin{array}{l}\text { How often did you have stomach contents (liquid or food) moving upwards to your } \\
\text { throat or mouth (regurgitation)? }\end{array}$ & 0 & 1 & 2 & 3 \\
\hline 3. & How often did you have a pain in the center of the upper stomach? & 3 & 2 & 1 & 0 \\
\hline 4. & How often did you have nausea? & 3 & 2 & 1 & 0 \\
\hline 5. & $\begin{array}{l}\text { How often did you have difficulty getting a good night's sleep because of your } \\
\text { heartburn and/or regurgitation? }\end{array}$ & 0 & 1 & 2 & 3 \\
\hline 6. & $\begin{array}{l}\text { How often did you take additional medication for your heartburn and/or regurgitation } \\
\text { other than what the physician told you to take (such as Maalox)? }\end{array}$ & 0 & 1 & 2 & 3 \\
\hline
\end{tabular}

Table 2: *Chi-square $\mathrm{p}$ value is $<0.001 .,{ }^{* *}$ chi-square $\mathrm{p}$ value is 0.0341 .

\begin{tabular}{|c|c|c|c|}
\hline Type of operation & Number/percentage of patients & $\begin{array}{c}\text { Number of patients persist to } \\
\text { Number of symptomatic GERD } \\
\text { patients before the operation } \\
\text { have GERD symptoms after the } \\
\text { operations }\end{array}$ & 42 \\
\hline Sleeve Gastrectomy & $218(30.4 \%)$ & 41 & $39(92.9 \%)^{*}$ \\
\hline Roux en-Y gastric bypass & $61(8.5 \%)$ & 98 & $7(17.1 \%)$ \\
\hline
\end{tabular}




\begin{tabular}{|c|c|c|c|}
\hline LAGB & $175(24.4 \%)$ & 56 & $20(35.7 \%)$ \\
\hline Total & 718 & 236 & $96(40.7 \%)^{* *}$ \\
\hline
\end{tabular}

Table 3: * chi-square $\mathrm{p}$ value is $<0.001,{ }^{* *}$ Chi-square $\mathrm{p}$ value is 0.0341 .

\begin{tabular}{|c|c|c|}
\hline Type of operation & $\begin{array}{c}\text { Number of patients with asymptomatic reflux } \\
\text { at contrast swallow study }\end{array}$ & $\begin{array}{c}\text { Number of these patients with New onset GERD } \\
\text { symptoms after the operation }\end{array}$ \\
\hline Sleeve Gastrectomy & 23 & $19(82.6 \%)^{*}$ \\
\hline Roux en-Y gastric bypass & 33 & $18(54.4 \%)$ \\
\hline One anastomosis gastric bypass & 28 & $15(53.6 \%)$ \\
\hline LAGB & 29 & $19(65.5 \%)$ \\
\hline Total & 113 & $71(62.8 \%)^{* *}$ \\
\hline
\end{tabular}

Table 4: *chi-square $p$ value is $=0.0131$.

\begin{tabular}{|c|c|c|}
\hline Type of operation & Number of patients & Symptomatic GERD after the operation total \\
\hline Sleeve Gastrectomy & $218(30.4 \%)$ & $87(39.9 \%)^{*}$ \\
\hline Roux en-Y gastric bypass & $61(8.5 \%)$ & $10(16.4 \%)$ \\
\hline One anastomosis gastric bypass & $264((36.8 \%)$ & $29(11 \%)$ \\
\hline LAGB & $175(24.4 \%)$ & $41(23.4 \%)$ \\
\hline Total & 718 & $167(23.3)$ \\
\hline
\end{tabular}

Our protocol that all patients should pass contrast swallow study before the surgery $[10,11]$, we examined which patients had a pre-operative asymptomatic reflux at contrast swallow study and a total of 113 patients out of $718(15.7 \%)$ had a positive swallow test (mild, moderate of severe) and of these patient 71 (62.8\%) developed GERD symptoms post-operatively without correlation to the degree of reflux at the swallow test but with statistically significant correlation to the type of operation especially for SG (P value <0.001) (Table 3) and to our knowledge this was never reported in the literature. There was a significant difference between the type of the bariatric procedure and the incidence of GERD symptoms after the operation. The incidence of symptomatic GERD in patients who underwent SG was 39.9\% ( $p=0.0131$ ). This was significantly higher comparing to $16.4 \%$ following roux en y gastric bypass, $23.4 \%$ following LAGB, and 11\% following OAGB (Table 4). There was no correlation between the incidence of GERD and; BMI at surgery; change in BMI; co-morbidities such as diabetes, hyperlipidemia, hypertension, and COPD (Table 5), we also examined which of the symptomatic per-operative GERD patients persist to have symptoms and we found that $92.9 \%$ of the patients that underwent SG with diagnosis of GERD pre-operatively persist to have symptoms after the operation (Table 2).

Table 5: Medical history and GERD after operation.

\begin{tabular}{|c|c|c|c|}
\hline & & Total & GERD after surgery \\
\hline \multirow{2}{*}{ Smoker } & Yes & 231 & $58(25.1 \%)$ \\
& No & 487 & $109(22.4 \%)$ \\
\hline \multirow{2}{*}{ Dyslipidemia } & Yes & 156 & $42(26.9 \%)$ \\
& No & 562 & $125(22.2 \%)$ \\
\hline \multirow{2}{*}{ Diabetes mellitus } & Yes & 161 & $34(21.1 \%)$ \\
& No & 557 & $111(19.9 \%)$ \\
\hline
\end{tabular}

\begin{tabular}{|c|c|c|c|}
\hline \multirow{2}{*}{ Hypertension } & Yes & 193 & $46(23.8 \%)$ \\
& No & 525 & $121(23 \%)$ \\
\hline \multirow{2}{*}{ IHD } & Yes & 21 & $7(33.3 \%)$ \\
& No & 697 & $160(23 \%)$ \\
\hline \multirow{2}{*}{ IBD } & Yes & 4 & $1(25 \%)$ \\
\cline { 3 - 4 } & No & 714 & $166(23.2 \%)$ \\
\hline \multirow{2}{*}{ COPD } & Yes & 31 & $6(19.4 \%)$ \\
& No & 687 & $161(23.4 \%)$ \\
\hline \multirow{2}{*}{ Cirrhosis } & Yes & 5 & $1(20 \%)$ \\
& No & 713 & $166(23.3 \%)$ \\
\hline \multirow{2}{*}{ Renal Failure } & Yes & 2 & $0(0 \%)$ \\
& No & 716 & $167(23.3 \%)$ \\
\hline
\end{tabular}

\section{Discussion}

We found that $23.3 \%$ of our patients persist to have or developed symptomatic GERD symptoms after the bariatric operation. We found a significant difference between the type of the bariatric procedure and the risk of GERD with the highest prevalence after LSG (39.9\%) compared to $11 \%$ after OAGB, $23.4 \%$ after LAGB, and $16.4 \%$ after RNYGB, respectively. Of these patients 159 patients responds well to PPI and 8 patients needed further investigation with contrast swallow and gastroscopy and of them 2 patients operated for hiatal hernia repair, one after sleeve gastrectomy and the other after OAGB. Our findings is consistent with some previous studies as Howard et al [12], Frezza et al [13], however our finding that smoking is not related to symptomatic GERD is inconsistent with Kohata et al which said smoking cessation reduced the prevalence of GERD and the severity of reflux [14], but in our study only bariatric patients examined which is not the same population in other studies about relation between smoking and GERD. We think 
according to our results that every patient should be questioned for GERD symptoms before the operation. Sleeve gastrectomy should be contraindicated in bariatric patients with symptoms of GERD or in asymptomatic patients with reflux on contrast swallow study. Moreover, although currently major guidelines make no definite statement about sleeve gastrectomy in patients with asymptomatic reflux [15], our findings are not correlated with Petersen WV et al that states SG significantly increased lower esophageal pressure and may protect obese patients from gastro-esophageal reflux [16], however they are consistent with Stenard F, et al [15].

None of the parameters in our study apart from the type of the bariatric surgery, symptoms of GERD before the operation and reflux at the contrast study were found to be of value in predicting the development of GERD postoperatively. The limitations of our study include its single-center focus, the relatively small number of SG patients, and the retrospective study design.

\section{Conclusion}

Sleeve gastrectomy is a good bariatric procedure option but should be contraindicated in asymptomatic reflux contrast swallow study and symptomatic GERD patients preoperatively because of high levels of symptomatic GERD post-operatively. Asymptomatic reflux at contrast swallow study pre-operatively should be considered a risk factor for GERD after the operation.

\section{References}

1. Lemaitre F, Léger P, Nedelcu M, Nocca D (2016) Laparoscopic sleeve gastrectomy in the South Pacific. Retrospective evaluation of 510 patients in a single institution. Int J Surg 30: 1-6.

2. Iannelli A, Dainese R, Piche T, Facchiano E, Gugenheim J (2008) Laparoscopic sleeve gastrectomy for morbid obesity. World Gastroenterol 14(6): 821-827.

3. Sneineh MA, Harel L, Elnasasra A, Razin H, Rotmensh A, et al. (2020) Increased Incidence of Symptomatic Cholelithiasis After Bariatric RouxEn-Y Gastric Bypass and Previous Bariatric Surgery: a Single Center Experience. Obes Surg 30(3): 846-850.

\section{ISSN: 2574-1241}

DOI: 10.26717/BJSTR.2020.31.005153

Midhat Abu Sneineh. Biomed J Sci \& Tech Res

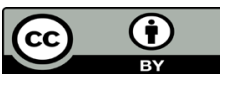

This work is licensed under Creative Commons Attribution 4.0 License

Submission Link: https://biomedres.us/submit-manuscript.php
4. Mustafa El Hadi, Daniel W Birch, Richdeep S Gill, Shahzeer Karmali (2014) The effect of bariatric surgery on gastroesophageal reflux disease. Can J Surg. 57(2): 139-144.

5. Orlando RC (2001) Overview of the mechanisms of gastroesophageal reflux. Am J Med 111 Suppl 8A: 174S-177S.

6. Suter M, Dorta G, Giusti V, Calmes JM (2004) Gastro-esophageal reflux and esophageal motility disorders in morbidly obese patients. Obes Surg 14(7): 959-966.

7. El Hadi M, Birch DW, Gill RS, Karmali S (2014) The effect of bariatric surgery on gastroesophageal reflux disease. Can J Surg 57(2): 139-144.

8. Suzuki H, Matsuzaki J, Okada S, Hirata K, Fukuhara S, et al. (2013) Validation of the GerdQ questionnaire for the management of gastrooesophageal reflux disease in Japan. United European Gastroenterology Journal 1(3): 175-183.

9. Jones R, Junghard O, Dent J, N Vakil, K Halling, et al. (2009) Development of the GerdQ a tool for the diagnosis and management of gastrooesophageal reflux disease in primary care. Aliment Pharmacol Ther 30(10): 1030-1038.

10. Katz PO, Gerson LB, Vela MF (2013) Guidelines for the diagnosis and management of gastroesophageal reflux disease. Am J Gastroenterol 108(3): 308-328.

11. Lemanowicz A, Serafin Z (2014) Imaging of patients treated with bariatric surgery. Pol J Radiol 79: 12-19.

12. Howard DD, Caban AM, Cendan JC, Ben David K (2011) Gastroesophageal reflux after sleeve gastrectomy in morbidly obese patients. Surg Obes Relat Dis 7(6): 709-713.

13. Frezza EE, Ikramuddin S, Gourash W, T Rakitt, A Kingston, et al. (2002) Symptomatic improvement in gastroesophageal reflux disease (GERD) following laparoscopic Roux-en-Y gastric bypass. Surg Endosc 16(7): 1027-1031.

14. Kohata Y, Fujiwara Y, Watanabe T, Masanori Kobayashi, Yasuhiko Takemoto et al. (2016) Long-Term Benefits of Smoking Cessation on Gastroesophageal Reflux Disease and Health-Related Quality of Life. PLoS One 11(3): e0150554.

15. Stenard F, Iannelli A (2015) Laparoscopic sleeve gastrectomy and gastroesophageal reflux. World J Gastroenterol 21(36): 10348-10357.

16. Petersen WV, Meile T, Küper MA, Zdichavsky M, Königsrainer A, et al. (2012) Functional importance of laparoscopic sleeve gastrectomy for the lower esophageal sphincter in patients with morbid obesity. Obes Surg 22(3): 360-366.

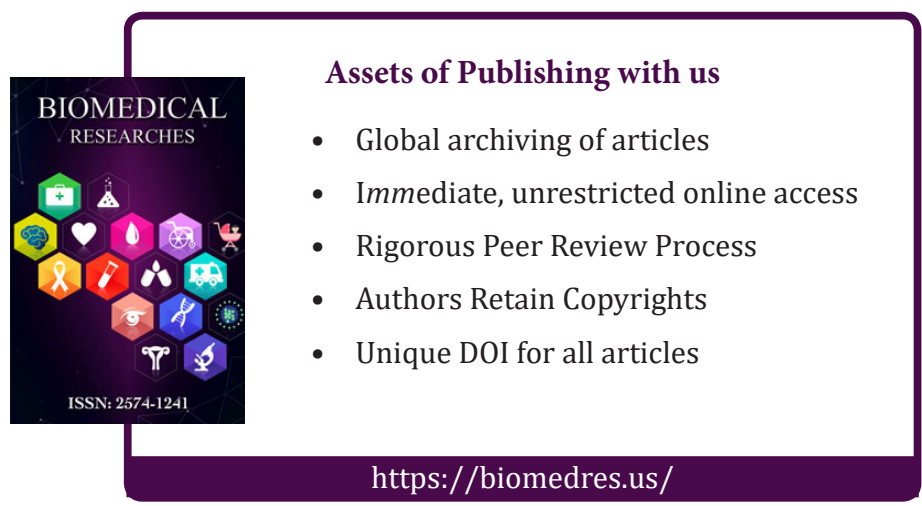

Copyright@ Midhat Abu Sneineh | Biomed J Sci \& Tech Res | BJSTR. MS.ID.005153. 